\title{
Situs inversus totalis EM RATO WISTAR
}

\author{
CARNEVALI, Taiane Rita ${ }^{1}$; \\ GIORDANI, Cláudia ${ }^{2}$; \\ MATOS, Caroline Bohnen de ${ }^{2}$; \\ GUTERRES, Karina Affedlt ${ }^{2}$; \\ SILVA, Cristine Cioato da ${ }^{2}$; \\ CLEFF, Marlete Brum ${ }^{3}$.
}

${ }^{1}$ Médica Veterinária, Mestre, Universidade Federal de Pelotas; ${ }^{2}$ Doutoranda do Programa de Pós-Graduação em Veterinária, Universidade Federal de Pelotas; ${ }^{3}$ Professora, Doutora, Departamento de Clínicas Veterinárias, Faculdade de Veterinária, Universidade Federal de Pelotas.

\section{RESUMO}

Citus inversus, uma alteração rara na qual os órgãos se desenvolvem no lado oposto de sua localização habitual, já foi relatada em diferentes espécies. Seu diagnóstico é baseado nos exames por imagem, sendo geralmente um achado ocasional. Diante da raridade da alteração, o objetivo foi descrever a ocorrência de Situs inversus totalis em um animal experimental, por meio de exame radiográfico e de necropsia. Uma rata albina Wistar, com 16 semanas, utilizada para estudo experimental independente, foi submetida à radiografia torácica e abdominal, sendo observada diferente disposição dos órgãos internos. Após o término do experimento, procedeu-se a necropsia do animal, em que foi observada a localização oposta à anatômica dos órgãos: fígado, baço, estômago, ceco, pulmão e coração, caracterizando Situs inversus totalis com dextrocardia.

Palavras-chave: Veterinária. Órgãos. Assimetria. Dextrocardia. 


\section{INTRODUÇÃO}

A alteração congênita Situs inversus é considerada rara, pouco relatada nos mamíferos, sendo definida pelo desenvolvimento dos órgãos no lado oposto de sua anatomia fisiológica (JERRAM et al., 2006; SALMAN et al., 2010; SHIRAIWA et al., 1995; SOUZA JUNIOR et al., 2011). Existem relatos de Situs inversus em uma gama imensa de espécies, entre elas, humanos, animais domésticos e silvestres (BAKER-COHEN, 1961; EVANS, 1987; JERRAM et al., 2006; KAYA et al., 2002; LARSEN; KIRK, 1987; NEIL et al., 2002; PINHEIRO et al., 2014; RIES; KÖNIG, 1988; SHIRAIWA et al., 1995; TURNER; JONES, 2004). A ocorrência desta alteração corresponde a 0,01-0,2\% em ratos e 0,01-0,02\% em humanos (JERRAM et al., 2006; SHIRAIWA et al., 1995), sem predisposição sexual (WILHELM; HOLBERT, 2015) e geralmente assintomática (SALMAN et al., 2010).

A alteração é classificada em totalis quando a posição das vísceras é totalmente invertida da usual, e partialis quando algumas estruturas estão parcialmente invertidas ou em posição anormal (SHIRAIWA et al, 1995). Além disso, também há uma classificação para o posicionamento anômalo do coração, sendo Situs inversus com levocardia quando o ápice cardíaco aponta para o lado esquerdo da linha média, e com dextrocardia quando ocorre para o lado direito (CASANOVA et al., 2006).

De acordo com a literatura, o Situs inversus pode ser observado como alteração única ou associado à Síndrome de Kartagener, caracterizada pelo Situs inversus, pansinusite crônica e bronquiectasias (KAYA et al., 2002; SHIRAIWA et al., 1995; SWENSSON et al., 2003; WITSBERGER et al., 2009), que resulta na disfunção da motilidade de cílios e flagelos (discinesia ciliar primária) (SOUZA JUNIOR et al., 2011).

O diagnóstico em geral é obtido através de exames de imagem, achados de cirurgias e ou necropsias e a conduta terapêutica remete-se apenas às alterações ocasionadas pela discinesia ciliar (HAWKINS, 2001; MEDEIROS SOBRINHO et al., 1986; SOUZA JUNIOR et al., 2011).

Diante da raridade de diagnóstico desta alteração em animais experimentais, o objetivo deste trabalho foi relatar um caso de Situs inversus totalis (SIT) com dextrocardia em rato albino Wistar. 


\section{RELATO DE CASO}

Um grupo de ratos foi incluído como modelo experimental em trabalho de pesquisa, registrado na Comissão de Ética em Experimentação Animal (CEEA) da Universidade Federal de Pelotas (UFPEL) sob o $n$ ㅇ 6538. Durante duas semanas, antes da avaliação, todos os animais foram adaptados ao manuseio para o desenvolvimento da pesquisa. Após esse período, uma rata albina, linhagem Wistar, com quatro meses de idade, $230 \mathrm{~g}$, foi submetida a exame radiográfico para avaliação do trânsito gastrointestinal, como uma etapa do experimento.

Ao término do experimento o animal foi submetido à eutanásia conforme a Resolução no 1000 do Conselho Federal de Medicina Veterinária (CFMV, 2012) e, à necropsia, confirmouse o diagnóstico de SIT.

\section{RESULTADOS E DISCUSSÃO}

Durante o experimento, somente um animal apresentou imagens radiográficas com disposição dos órgãos diferentes dos demais, sendo diagnosticado com Situs inversus totalis, e dentro do grupo experimental $(n=48)$ o achado representou 2,08\%, uma média elevada quando comparada aos dados de literatura (SALMAN et al., 2010; SHIRAIWA et al., 1995). Quando analisados os dados do Biotério Central (fundado na década de 80), local onde os animais foram adquiridos (produção média mensal de 500 ratos Wistar), esta alteração ainda não havia sido relatada (registros do Biotério Central-UFPEL).

No exame radiográfico do animal experimental constatou-se: ápice cardíaco voltado para a direita e base cardíaca voltada para a esquerda (dextrocardia), assim como estômago do lado direito da cavidade abdominal, com piloro voltado para a esquerda (Figura 1), não sendo visualizadas demais alterações radiográficas. 


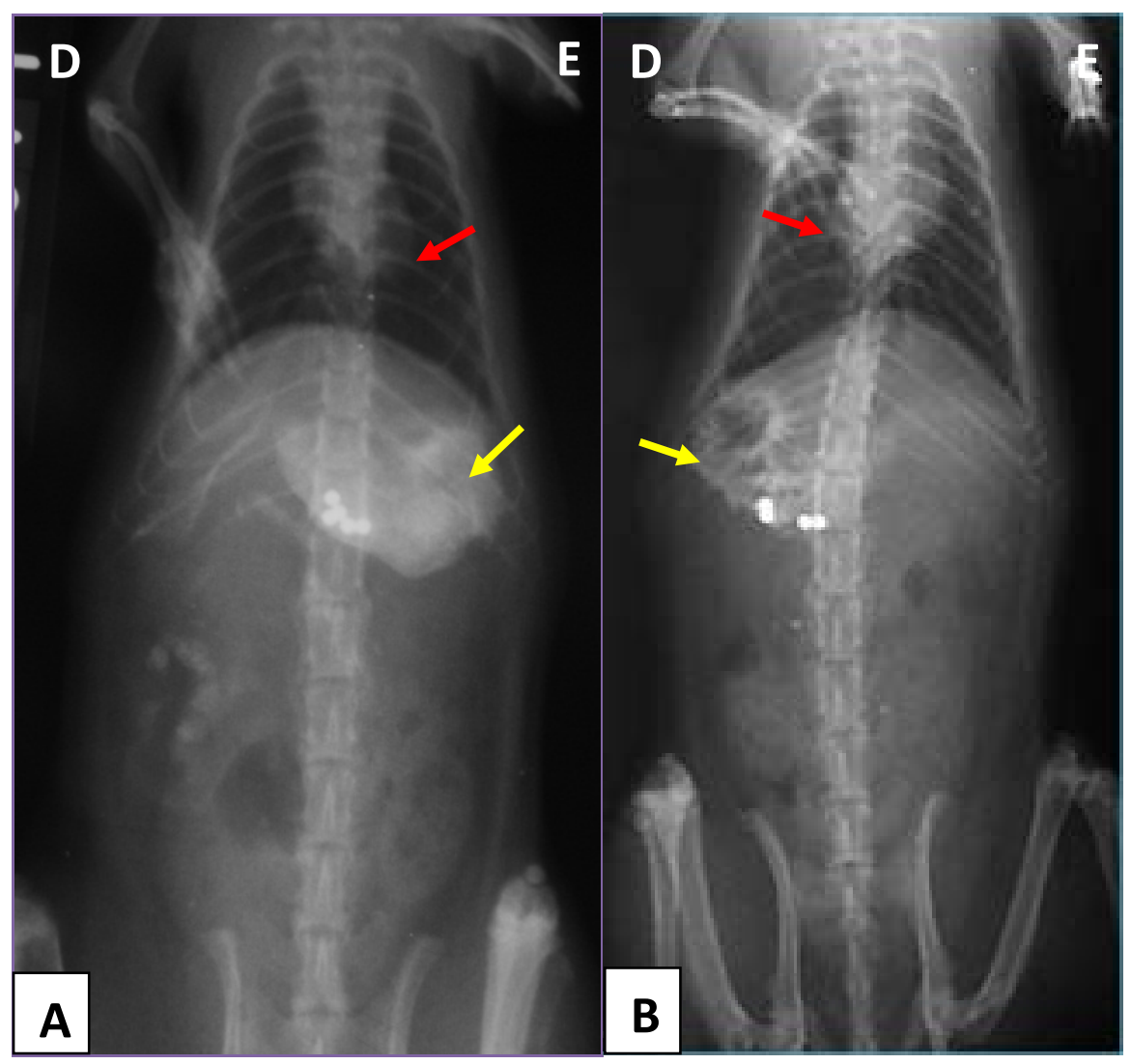

Figura 1 - Imagens radiográficas de tórax e abdômen em projeção ventrodorsal de um rato Wistar normal (A), e de uma rata Wistar com Situs inversus totalis (B). Base cardíaca (seta vermelha) e estômago (com esferas metálicas e bário em seu interior; seta amarela). Letras indicando os lados direito (D) e esquerdo (E) do animal.

Segundo Souza Junior et al. (2011), os exames por imagem, como radiografias e ultrassonografias, são considerados de eleição para a identificação de Situs inversus, porém devido sua raridade, deve-se ter uma maior atenção no exame para a identificação da anormalidade. A descoberta dessa alteração revela-se de extrema importância, mesmo que assintomática, principalmente nos pacientes que passarão por procedimento invasivo e/ou cirúrgico, por alterar a anatomia normal e consequentemente influenciar na técnica dos procedimentos (CAHUA et al., 2015; WITSBERGER et al., 2009).

O animal experimental não apresentou nenhuma anormalidade clínica durante o período em que esteve em observação no estudo, concordando com a literatura, que relata a ausência de efeitos adversos sobre a saúde do indivíduo na maioria dos casos (SALMAN et al., 2010). Quando presentes, os sinais clínicos são geralmente devido à discinesia ciliar primária, apresentando sinais respiratórios que variam de rinossinusite até alterações pulmonares, 
podendo cursar com hidrocefalia, otite, e até sub ou infertilidade (NEIL et al., 2002; REICHLER et al., 2001; RODRIGUES et al., 2008; SOUZA JUNIOR et al., 2011; STOWATER, 2005). Como manejo terapêutico da síndrome deve-se evitar a obesidade, irritantes das vias aéreas e a agitação por tempo prolongado; além da utilização de broncodilatadores para facilitar a respiração e antibioticoterapia para combater infecções secundárias (SOUZA JUNIOR et al., 2011).

Na necropsia, ao exame da cavidade abdominal observou-se a alteração na localização dos órgãos: fígado e ceco (lado esquerdo) e baço junto ao estômago (lado direito). Já na avaliação da cavidade torácica observou-se o pulmão rotado para o lado direito do tórax e ápice cardíaco voltado para a direita e a base cardíaca para o lado esquerdo (Figura 2).

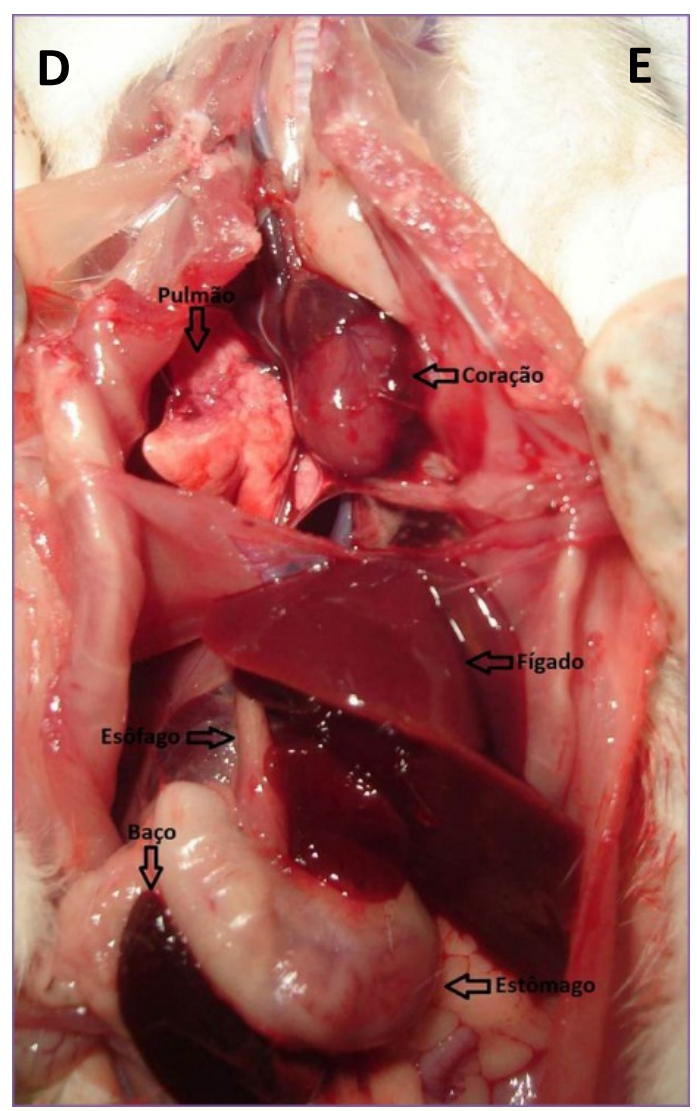

Figura 2 - Animal experimental com Situs inversus totalis, em decúbito dorsal, durante necropsia, demonstrando a localização inversa dos órgãos da cavidade torácica e abdominal (setas). Letras indicando os lados direito (D) e esquerdo (E) do animal.

A partir dos resultados obtidos no exame radiográfico e confirmados através da necropsia, estabeleceu-se o diagnóstico de Situs inversus totalis (SIT) com dextrocardia, sendo que as 
alterações encontradas no animal são as descritas em outros estudos de SIT (JERRAM et al., 2006; SALMAN et al., 2010; SHIRAIWA et al., 1995). Mesmo havendo casos esporádicos, existem evidências da herança autossômica recessiva da lateralidade de defeitos em humanos (ARNOLD et al., 1983; CHIB et al., 1977; ZLOTOGORA et al., 1987). Ainda hoje, a causa definitiva do SIT não está elucidada, e o mecanismo de ocorrência tem teorias na embriogênese (CAHUA et al., 2015). Uma das teorias cita que os cílios são responsáveis pela rotação dos órgãos internos, e que devido seu funcionamento incorreto, isso não ocorreria (AFZELIUS, 1995). Segundo Vogel et al. (2010), dois tipos de cílios no nó embrionário determinam a assimetria esquerda-direita, os cílios móveis (fluxo fluido para a esquerda) e os cílios mecanosensoriais (respondem ao fluxo). Por outro lado, Ryan et al. (1998) relatam a existência de genes que expressam assimetria, determinando o eixo direito/esquerdo, e Bartoloni et al. (2002) remetem a mutação do gene DNAH11 como envolvido na síndrome de Situs inversus totalis.

\section{CONCLUSÃO}

Este relato reflete a importância do diagnóstico de Situs inversus totalis, pois em animais experimentais podem ocorrer erros nos resultados de pesquisa devido à presença dessa alteração congênita. A identificação e a exclusão desses animais da reprodução são essenciais em razão da herdabilidade genética. O diagnóstico na medicina veterinária permite o tratamento precoce, caso necessário, e consequentemente melhor qualidade de vida para esses animais. 


\section{Situs inversus totalis IN WISTAR RAT}

\section{ABSTRACT}

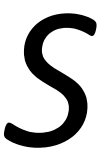

itus inversus is a rare change that causes the development of the organs on the opposite side of its usual location and it is reported in different species. Diagnosis is based on imaging tests, usually being an occasional finding. Due to the rarity of the change, the objective was to describe the occurrence of Situs inversus totalis in an experimental animal, using radiographic and necropsy examinations. A Wistar albino rat, 16 weeks of age, used for an independent experimental study, after thoracic and abdominal radiograph presented different arrangement of internal organs. After the experiment, the animal was submitted to necropsy, and it was observed the opposite location to the anatomical organs: liver, spleen, stomach, cecum, lung and heart; featuring Situs inversus totalis with dextrocardia.

Keywords: Animal. Organs. Asymmetry. Dextrocardia.

\section{Situs inversus totalis EN RATA WISTAR}

\section{RESUMEN}

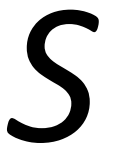

itus inversus es una rara enfermedad en la cual el desarrollo de los órganos ocurre en el lado opuesto a la habitual. Esta enfermedad se ha reportado en diferentes especies. El diagnóstico se basa en las pruebas de imagen, siendo por lo general, un hallazgo ocasional. Debido a la rareza de la alteración, el objetivo fue describir la ocurrencia de Situs inversus totalis en un animal de experimentación mediante examen radiográfico y la necropsia. Una rata Wistar albino, a las 16 semanas, que se utiliza para el estudio experimental independiente, después de radiografía torácica y abdominal se observó la disposición diferente de los órganos internos. Después del experimento, se procedió a la necropsia del animal, donde se observó la posición opuesta a la anatomía de los órganos: hígado, bazo, estómago, ciego, pulmón y corazón; caracterizando así el Situs inversus totalis con dextrocardia.

Palabras clave: Animales. Órganos. Asimetría. Dextrocardia. 


\section{REFERÊNCIAS}

AFZELIUS, B. A. Situs inversus and ciliary abnormalities. What is the connection? Journal of Developmental Biology, v. 39, n. 5, p. 839-844, 1995.

ARNOLD, G. L.; BIXLER, D.; GIROD, D. Probable autosomal recessive inheritance of polysplenia, Situs inversus and cardiac defects in an Amish family. American Journal of Medical Genetics, v. 16, n. 1, p. 35-42, 1983.

BAKER-COHEN, K. F. Visceral and vascular transposition in fishes, and a comparison with similar anomalies in man. American Journal of Anatomy, v. 109, n. 1, p. 37-55, 1961.

BARTOLONI, C.; BLOUIN, J.; PAN, Y.; et al. Mutations in the DNAH11 (axonemal heavy chain dynein type 11) gene cause one form of Situs inversus totalis and most likely primary ciliary dyskinesia. Proceedings of the National Academy of Science of the United States of America, v. 99, n. 16, p. 10282-10286, 2002.

CAHUA, J.; DIAS, D.; GONZALES-VIEIRA, O. Complete Situs inversus in 2 asymptomatic dogs. Topical in Companion Animal Medicine, v. 30, n. 1, p. 68-71, 2015.

CASANOVA, M. S.; TUJI, F. M.; YOO, H. J.; et al. Kartagener syndrome. Dentomaxillofacial Radiology, v. 35, n. 5, p. 386-389, 2006.

CFMV-CONSELHO FEDERAL DE MEDICINA VETERINÁRIA. Resolução $n^{\circ} 1000$ de 11 de maio de 2012. In: Manual de Legislação do Sistema CFMV/CRMVs, Módulo II: Ética e Profissões. Disponível em: <http://portal.cfmv.gov.br/portal/lei/download-arquivo/id/325>.

CHIB, P.; GROVER, D. N.; SHAHI, B. N. Unusual occurrence of dextrocardia with Situs inversus in succeeding generations of a family. Journal of Medical Genetics, v. 14, n. 1, p. 30-32, 1977.

EVANS, H. E. Cyclopia, Situs inversus and widely patent ductus arteriosus in a new-born pig, Sus scrofa. Anatomia Histologia Embryologia, v. 16, n. 3, p. 221-226, 1987.

HAWKINS, E. C. Distúrbios respiratórios. In: NELSON, R. W.; COUTO, C. G. Fundamentos de Medicina Interna de Pequenos Animais, Rio de Janeiro, p. 164-271, 2001.

JERRAM, R. M.; WARMAN, C. G. A.; CLEMENT, T. C. W. U. Echocardiographic and radiographic diagnosis: complete situs inversus in a cat. Veterinary Radiology Ultrasound, $v$. 47, n. 3, p. 313-315, 2006.

KAYA, A.; KAYA, S. U.; FITÖZ, S.; et al. Kartagener's syndrome. Turkish Respiratory Journal, v. 3, p. 113-116, 2002. 
LARSEN, C.; KIRK, E. J. Abdominal Situs inversus in a sheep. New Zealand Veterinary Journal, v. 35, n. 7, p. 113-114, 1987.

MEDEIROS SOBRINHO, J. H.; KAMBARA, A. M.; SILVA, M. V. D.; et al. Radiodiagnóstico das malposições cardíacas e cardioviscerais: apresentação de 100 casos. Arquivos Brasileiros de Cardiologia, v. 46, n. 6, p. 387-394, 1986.

NEIL, J. A.; CANAPP, S. O. J.; COOK, C. R.; et al. Kartagener's Syndrome in a Dachshund Dog. Journal of the American Animal Hospital Association, v. 38, p. 45-49, 2002.

PINHEIRO, V. L. C.; PEREIRA, L. C.; LIMA, A. R.; et al. Dextrocardia with situs solitus and inversion apex-basis axis in lesser anteater (Tamandua tetradactyla) - case report. Arquivo Brasileiro de Medicina Veterinária e Zootecnia, v. 66, n. 1, p. 116-120, 2014.

REICHLER, I. M.; HOERAUF, A.; GUSCETTI, F.; et al. Primary ciliary dyskinesia with Situs inversus totalis, hydrocephalus internus and cardiac malformations in a dog. Journal of Small Animal Practice, v. 42, p. 345-348, 2001.

RIES, R.; KÖNIG, H. E. Situs inversus of the lungs, heart and liver in a bull. Tierärztliche Prax, v. 16, n. 3, p. 251-252, 1988.

RODRIGUES, J. C.; OLM, M. A. K.; ADDE, F. V.; et al. Caso clínico: síndrome de Kartagener. Pediatria (São Paulo), v. 30, n. 1, p. 66-70, 2008.

RYAN, A. K.; BLUMBERG, B.; RODRIGUEZ-ESTEBAN, C.; et al. Pitx2 determines left-right asymmetry of internal organs in vertebrates. Nature, v. 394, p. 545-551, 1998.

SALMAN, B.; TEZCANER, T.; EGE, B.; et al. Situs inversus totalis in a Wistar albino rat. Journal of Veterinary Medicine and Animal Health, v. 2, n. 1, p. 6-7, 2010.

SHIRAIWA, K.; WATANABE, A; SATO N.; et al. Case report on Situs inversus totalis in two sprague-dawley rats. Experimental Animals, v. 44, n. 4, p. 341-345, 1995.

SOUZA JUNIOR, P.; SILVA, S. S. R.; MARTINS, M. C. Síndrome de kartagener em um cão (Canis lupus familiaris) da raça Cocker Spaniel Inglês. Arquivo Brasileiro de Medicina Veterinária e Zootecnia, v. 63, n. 3, p. 768-772, 2011.

STOWATER, J. L. Kartagener's syndrome in a dog. Veterinary Radiology Ultrasound, v. 17, p. 174-177, 2005.

SWENSSON, R. C.; JORGE JUNIOR, J. J.; SWENSSON, R. P. Síndrome de Kartagener relato de caso. Revista Brasileira de Otorrinolaringologia, v. 69, n. 6, p. 857-861, 2003.

TURNER, S. I.; JONES, R. M. Complete Situs inversus in a horse. Veterinary Record, v. 155, n. 3, p. 96, 2004. 
WILHELM, A.; HOLBERT, J. M. Situs inversus imaging. Medscap, nov. 2015. Disponível em: <http://emedicine.medscape.com/article/413679-overview>. Acesso em: 12 de dezembro de 2015.

WITSBERGER, T.; DISMUKES, D.; KELMER, E. Situs inversus totalis in a dog with a chronic diaphragmatic hernia. Journal of the American Animal Hospital Association, v. 45, n. 5, p. 245-248, 2009.

VOGEL, P.; READ, R.; HANSEN, G. M.; FREAY, L. C.; ZAMBROWICZ, B. P.; SANDS, A. T. Situs Inversus in $\mathrm{Dpcd} / \mathrm{Poll}^{-1}, \mathrm{Nme}^{-1}$, and $\mathrm{Pkd} 1 / 1^{-/-}$Mice. Veterinary Pathology, v. 47, n. 1, p. 120-131, 2010.

ZLOTOGORA, J.; SCHIMMEL, M. S.; GLASER, Y. Familial Situs inversus and congenital heart defects. American Journal of Medical Genetics, v. 26, n. 1, p. 181-184, 1987.

Autor para correspondência Marlete Brum Cleff. Universidade Federal de Pelotas, Faculdade de Veterinária, Campus Universitário, Capão do Leão (RS), Brasil. marletecleff@gmail.com 